\title{
Cerebral venous volume changes and pressure autoregulation in critically ill infants: an editorial comment
}

\author{
Frank van Bel ${ }^{1} \cdot$ Gunnar Naulaers $^{2}$
}

Received: 13 February 2020 / Revised: 24 February 2020 / Accepted: 26 February 2020 / Published online: 10 March 2020

(c) Springer Nature America, Inc. 2020

The article of Govidan et al. in this issue of the Journal of Perinatology [1] highlights the role of ventilator-related changes in cerebral (venous) blood volume on cerebral pressure passivity, possibly predisposing to brain damage, in a heterogenous group of neonates subjected to positivepressure ventilation. This study brought up an important issue and actually elaborates in some way on studies done in the eighties of the last century by Perlman et al. in which muscle paralysis was used to decrease the incidence of ventilator-induced cerebral perfusion swings in sick very preterm infants as investigated with Doppler ultrasound monitored cerebral blood flow velocities. It was shown that this policy substantially lowered the incidence of periventricular-Intraventricular hemorrhages (PIVH). Later on increasing efforts were done to replace positive-pressure ventilation by more sophicated noninvasive ventilation strategies to avoid (cerebral) hemodynamic pertubations [2]. The retrospective set-up of the Govidan paper prohibits to conclude that lack of cerebral autoregulation (cerebral pressure passivity) indeed added to additional brain damage in their investigated patient population, given its heterogenicity and incomplete covering of the near infrared spectroscopy monitored brain circulation during the very first hours and days of life. However, their studies provide further evidence that (longstanding) episodes of pressurepassive perfusion potentially lead to hypo- and hyperperfusion of the vulnerable immature brain.

Several studies performed in extremely and very preterm infants, partly already referenced by the authors, confirm

Frank van Bel

f.vanbel@umcutrecht.nl

1 Department of Neonatology, Wilhelmina Children's Hospital and Brain Center Rudolf Magnus, University Medical Center Utrecht, Utrecht University, Utrecht, The Netherlands

2 Department of Neonatology, University Hospitals Leuven, Leuven, Belgium that hyperperfusion with or without substantial blood pressure swings was "related" with an increased incidence of PIVH in patients with blood pressure passivity [3-5]. Also treatment with positive inotropics for supposed hypotension of prematurity may affect autoregulatory ability of the cerebral vascular bed and hence increase PIVH incidence [6]. Term infants suffering severe hypoxicischemic encephalopathy due to perinatal asphyxia are known to have often a pressure-passive (hyper)perfusion of the brain although a causal relation with additional brain damage is difficult to proof in this population. In a large single center study of our own group we found that up to $30 \%$ of the neonates had an impairment of autoregulatory ability during (noncardiac) major surgery (unpublished observations).

We agree with the authors that the role of pressure passivity is underestimated in the unstable and sick neonate and that noninvasive monitoring of the ability to autoregulate brain perfusion should be mandatory. We also support that this should be part of the standard care applied for this vulnerable group of patients. The next question is what is the most convenient way to attain this, for the patient as well as for the caregiver?

The authors used changes of $\mathrm{HbD}$ and $\mathrm{HbT}$ as a correlate for cerebral blood flow changes, a well validated bedside method. However, using this method, it remains difficult to measure autoregulation in a direct way. New measurement techniques (e.g., spatially resolved spectroscopy) and new signal analysis methods made it possible to measure cerebral autoregulation in a more convient and continuous way in the NICU, as described by two recent reviews [7, 8]. These techniques might be easier in use, although further validation is needed in larger studies. It is also important to develop well-defined indications to measure cerebral autoregulation as it is not necessary in the majority of the infants admitted to the NICU. It can be restricted to neonates with ventilation, low blood pressure, inotropic treatment, or other pharmacologic therapies possibly negatively affecting cerebral autoregulation or patients needing neonatal surgery. 
Randomized trials will be necessary to proof that monitoring cerebral autoregulatory ability will make a difference in brain development and neurological outcome.

\section{Compliance with ethical standards}

Conflict of interest The authors declare that they have no conflict of interest.

Publisher's note Springer Nature remains neutral with regard to jurisdictional claims in published maps and institutional affiliations.

\section{References}

1. Govindan V, Govindan R, Massaro AN, Al-Shargabi T, Andescavage NN, Vezina G, et al. Cerebral venous volume changes and pressure autoregulation in critically ill infants. J Perinatol. 2020; https://doi.org/10.1038/s41372-020-0626-0 (this issue).

2. Perlman JM, Goodman S, Kreusser KL, Volpe JJ. Reduction in intraventricular hemorrhage by elimination of fluctuating cerebral blood flow velocity in preterm infants with respiratory distress syndrome. N Engl J Med. 1985;312:1353-7.

3. Hoffman SB, Cheng YJ, Magder LS, Shet N. Cerebral autoregulation in premature infants during the first $96 \mathrm{~h}$ of life and relationship to adverse outcome. Arch Dis Child Fetal Neonatal Ed. 2018;104:F473-F479.

4. Vesoulis ZA, Flower AA, Zanelli S, Rambhia A, Abubakar M, Whitehead HV, et al. Blood pressure extremes and severe IVH in preterm infants. Pediatr Res. 2019;87:69-73.

5. Rhee CJ, Sortica da Costa C, Austin T, Brady KM, Czosnyka M, Lee JK. Neonatal cererbrovascular autoregulation. Pediatr Res. 2018;84:602-10.

6. Alderliesten T, Lemmers PMA, van Haastert IC, de Vries LS, Bonestroo HJC, Baerts W, et al. Hypotension in preterm neonates: low blood pressure alone does not affect neurodevelopmental outcome. J Pediatr. 2014;164:986-91.

7. Kooi EMW, Verhagen EA, Elting JWJ, Czosnyka M, Austin T, Wong FY, et al. Measuring cerebrovascular autoregulation in preterm infants using near-infrared spectroscopy: an overview of the literature. Expert Rev Neurother. 2017;17:801-18.

8. Thewissen L, Caicedo A, Lemmers P, Van Bel F, Van Huffel S, Naulaers G. Measuring near-infrared spectroscopy derived cerebral autoregulation in neonates: from research tool toward bedside multimodal monitoring. Front Pediatr. 2018;6:117. 